\title{
Germanica
}

GERMANICA $\quad 10 \mid 1992$

Mosaïques littéraires

\section{Peter Handke, une question de regard}

Das visuelle Moment bei Peter Handke

\section{Arlette Camion}

\section{OpenEdition}

Journals

Édition électronique

URL : http://journals.openedition.org/germanica/2100

DOI : 10.4000/germanica.2100

ISSN : 2107-0784

\section{Éditeur}

Université de Lille

\section{Édition imprimée}

Date de publication : 1 janvier 1992

Pagination : 155-168

ISSN : 0984-2632

\section{Référence électronique}

Arlette Camion, «Peter Handke, une question de regard », Germanica [En ligne], 10 | 1992, mis en ligne le 03 avril 2014, consulté le 06 octobre 2020. URL : http://journals.openedition.org/germanica/2100 ; DOI : https://doi.org/10.4000/germanica.2100

Ce document a été généré automatiquement le 6 octobre 2020.

(C) Tous droits réservés 


\title{
Peter Handke, une question de regard
}

\author{
Das visuelle Moment bei Peter Handke
}

\section{Arlette Camion}

1 Handke ne tient pas à raconter d'histoires. L'ambiguïté de son projet, donné dès le début, est toute entière dans l'exigence de récit (car il revendique, avec de plus en plus d'insistance, la position d' 'Erzähler») et le refus des prestiges et des illusions du roman. Son écriture répugne à épouser les formes de la narration, elle les rompt ou les force. Substituant au dire le geste de montrer, elle cherche à rendre visible ce qui ne l'était pas tout à fait, à déchiffrer ce qui était encore muet, elle est inscription davantage que discours. Voilà comment il définit l'activité d'écrire :

C'est comme lorsque, devant vous, vous avez une pierre dont vous ne pouvez déchiffrer l'inscription à l'œil nu. Vous posez alors une feuille de papier sur la pierre et vous y passez un crayon, de telle sorte qu'apparaît l'inscription, c'est-àdire l'écriture.

(Zr, 230)

2 Situant l'écriture d'emblée du côté de l'image, il lui demande, selon le mot de Klee, non « de rendre le visible mais de rendre visible »" . L'idéal serait pour elle de faire tableau, tout en acceptant sa destination première, qui est de faire récit. Elle allierait, en un point d'équilibre fragile, la statique de l'image et la dynamique du texte. Ce point d'équilibre n'est pas tout de suite atteint, ni toujours tenu. Les premiers livres font place nette, ils disloquent les structures obligées de la narration, malmènent l'intrigue, font éclater les éléments du texte. Il y est peu question du bonheur de voir, que Marianne dans La Femme gauchère et Keuschnig dans L'Heure de la sensation vraie sont les premiers à pressentir. Sorger dans Lent retour poursuit la recherche, en assumant la possibilité de son échec. Jusqu'à La Leçon de la Sainte-Victoire, il n'y a pas de certitudes, et les livres qui suivront sont eux aussi écrits dans l'espace du risque. Mais c'est précisément ce qui fait leur intérêt.

3 Bienvenue au conseil d'administration est un recueil de premiers textes qui sont autant de bouts d'essai. Ils ont en commun le refus de se clore en une histoire. Empruntant 
largement aux formes du cinéma, ce sont de fausses bandes-annonces, de faux synopsis, de faux scénarios. Récits au second degré, ils exhibent leur statut de simulacres et s'ouvrent sur le vertige d'une mise en abyme. L'imaginaire cinématographique n'est que la matrice d'un matériau neutre, images ayant perdu leur référent, oscillant entre vide et mythe, comme les inquiétantes Marilyn Monroe d'Andy Warhol. Le texte cesse abruptement, comme par une décision gratuite de l'auteur qui rompt une reproduction pouvant se poursuivre à l'infini. La langue froidement objective, que l'on a parfois rapproché de l'allemand de chancellerie de Kafka (mais Handke, à l'époque n'était-il pas étudiant en droit et fasciné par la dureté du langage juridique ?), cette volonté d'àplat renforce encore l'impression d'extériorité de ces images, données comme sur le support d'une pellicule. Dans Le Monde intérieur du monde extérieur du monde intérieur, dont le titre suffit à produire le malaise, Handke utilise les techniques expérimentales du collage et du ready-made, laconiquement exposé en poème. Radicalisant le refus du dire, l'objet trouvé s'auto-reproduit en se passant de la médiation du texte. Dès ces premiers livres, Handke puise dans les techniques du film des méthodes d'écriture. Ainsi par exemple le choix du hors-champ, qu'il utilisera jusqu'à L'Heure de la sensation vraie. Le petit récit La Guerre éclate est constitué de trois gros-plan fixes, montrant les mains d'un homme et d'une femme assis sur un banc, puis une porte battante ensuite plaquée contre un mur par le mouvement brutal d'une foule qui s'enfuit. C'est le moment où "la guerre éclate", hors image. Comme chez Faulkner, comme chez les écrivains du Nouveau Roman français, le récit est mis en marge, relégué aux franges de l'événement qui reste innommé, innommable. Chez Faulkner, il est l'abject, chez Robbe-Grillet, il est le pervers. Chez Handke, il est l'angoisse. Non pas celle de Roquentin dans La Nausée, une angoisse existentielle encore raisonnée, philosophique ; non pas l'étrange décalage dont est affecté le Meursault de Camus, mais quelque chose de primitif, de viscéral. Une panique inscrite dans le corps et pourtant sans objet précis, une épouvante qui pourrait être celle de la disparition. Le non-vu qui hante les premiers textes de Handke se laisserait de la sorte saisir : c'est l'éblouissement qui se produit au cours de cet événement inouï qu'est la disparition. L'absence, la mort sont encore explicables, la disparition ne l'est plus. Elle est brusque éclipse de ce qui, un instant auparavant, était encore là : la mère folle dans l'obscurité de la forêt $(\mathrm{kB}, 10)$, le paysage familier après le bombardement $(\mathrm{kB}, 9)$. Ainsi la modalité du brutal retrait des images est-elle la structure même du premier roman de Handke, Les Frelons. Alors que Claude Simon dans Orion aveugle n'introduit qu'un éblouissement passager, dû à la douleur physique qui fait éclater les perceptions en débris d'images, ici la cécité est définitive, et les images qui occupent toute la surface du récit, organisées en une suite désordonnée de séquences, n'ont plus comme centre le lieu assignable d'un regard. Ce sont des images sans sujet, des images qui disparaissent dans le trou noir d'un regard mort. Le lecteur ne sait plus d'où elles lui proviennent et elles peuvent être le matériau de diverses histoires: celle de quelqu'un qui se souvient du jour où il est devenu aveugle, celle de quelqu'un qui est aveugle et qui se souvient du jour où son frère s'est noyé, celle de quelqu'un qui se souvient d'un livre où quelqu'un devenait aveugle, où le frère de quelqu'un se noyait. Mais cela pourrait être aussi, comme dans Providence d'Alain Resnais, les images qui assaillent l'écrivain au travail, leur incessant bourdonnement de frelons ${ }^{2}$. Parfois les images se chevauchent, se brouillent. Toujours leur présence est intolérable, car rien ne peut la faire cesser, nul retour à un réel à jamais disparu. L'agressivité est latente dans les premiers textes. Elle est moins contenue dans la violence des images que dans leur autonomie : celui qui voit sans yeux 
devient la proie des choses, «des vibrations de souffrance qui sont dans les choses" $(H, 197)$. Lorsque, sous l'espèce d'un personnage nettement repérable, Josef Bloch dans L'Angoisse du gardien de but au moment du penalty, Handke réintroduit un lieu du regard, les images n'en sont pas pour cela maîtrisées : Bloch est moins sujet que victime de la perception. Il voit « les objets comme s'ils étaient leur propre réclame » (AdT, 52), dans un déferlement semblable à celui des signes publicitaires dans l'espace urbain, lancinants, péremptoires. Immobilisé dans la position du gardien de but, face à un danger qui ne se déclare pas, Bloch perçoit du monde des détails qui sont autant d'indices dont le sens est obscur. L'apparente linéarité du récit, qui permit à Wenders de le transposer si facilement à l'écran ${ }^{3}$, n'est le garant d'aucun ordre, au contraire, elle marque la progression du chaos. Handke est parvenu dans ce livre à rendre presqu' " optiquement » l'évolution vers la schizophrénie. Bloch passe insensiblement dans les images du monde, chacune d'entre elles l'évide un peu plus, et à mesure qu'il perd la possibilité de formuler avec les mots du discours les perceptions du monde, celles-ci gagnent du terrain, l'envahissent, jusqu'à ce que sujet et objet ne fassent plus qu'un continuum monotone qui est la figure de l'aliénation. Le schéma spatial reste celui de la frontalité, semblable au mode d'apparition des images pour le spectateur de cinéma, livré, passif. Avec cette volonté de tout voir, ce désir de panopticité propre à celui qui fréquente les salles obscures, et, en même temps l'incapacité à saisir un flot d'images qui restent étrangères. La conscience de Bloch « semble être un trou aveugle " (AdT, 62). Le thème de la cécité n'a pas disparu, il continuera à hanter les livres de Handke, jusqu'à La Leçon de la Sainte-Victoire où l'on le retrouvera sous sa dernière métamorphose : sous la forme de la " cécité aux couleurs ». Mais la cécité de Bloch est absence d'être, la cécité aux couleurs du narrateur-admirateur de Cézanne est une absence à l'être qu'il se reproche, intermittente, douloureuse parfois, pourtant nullement irrémédiable.

4 À partir de La courte lettre pour un long adieu, le récit de Handke s'achemine vers une positivité : au malaise que décrivent les premiers livres succède, plus ou moins avouée, la volonté de venir à bout des conflits et des contradictions. C'est pourquoi les déambulations, les périples et les dérives qui font la trame des «ciné-romans » des années soixante-dix ${ }^{4}$ peuvent être vus comme autant de Bildungsreisen. Handke les place lui-même sous les célèbres parrainages du Wilhelm Meister, de Karl Philip Moritz, de Gottfried Keller. Mais, comme ceux de Wim Wenders, les voyages de Handke ne mènent pas vraiment à l'insertion dans le monde des hommes, à la réconciliation avec lui. Dans le scénario de Faux mouvement, le nouveau Wilhelm Meister termine sa traversée de l'Allemagne seul, au sommet de la Zugspitze et décide là de devenir écrivain. Dans $A u$ fil du temps de Wenders, les deux personnages longent mélancoliquement la frontière qui déchire le pays, à la recherche à la fois des images simples et vraies d'un cinéma perdu (celui que le voyageur de La courte lettre pour un long adieu admire en John Ford), et d'un lieu où il y ait lieu d'être. Ils ne découvrent qu'un imaginaire "colonisé par les images des autres » et une langue qui a servi au mensonge. Le seul salut, c'est l'enfant: la dernière scène du film montre les deux pauvres cow-boys en version européenne sur le remblai d'une voie ferrée où un enfant, patiemment, leur réapprend les mots. Pour Handke dans Histoire d'enfant, le poids écrasant de l'Histoire ne peut être aboli que par cet autre regard, qui, à chaque nouvel enfant, remet tout en question. La dimension utopique des romans de Handke réside dans la radicalité de cet "autre regard». C'est le vide, délibérément ménagé par Marianne dans La Femme gauchère qui lui permet de reconstituer l'espace de sa vie ; c'est 
l'épreuve des lieux vierges du Grand Nord pour Sorger, dans Lent retour. Ainsi le schéma narratif est-il, davantage que celui du Bildungsroman, celui de la passion: mort et résurrection d'Andréas Loser dans Le Chinois de la douleur. Et cette renaissance se fait par une sorte de retournement perceptif. Si l'autre regard déjoue la cécité, dépasse le morcellement du monde en débris d'images, c'est qu'il est la subite révélation de l'unité spatiale. Dès Faux mouvement, Wilhelm parle d'une modalité tout à fait particulière de la perception qu'il nomme " regard érotique »:

Je sais que je n'ai pas ce que l'on nomme le don de l'observation mais j'ai par contre ou je me flatte d'avoir la faculté d'une sorte de regard érotique. Tout à coup je vois quelque chose que jusqu'alors je n'avais pas remarqué. A ce moment-là non seulement je le perçois, mais encore j'en éprouve quelque chose. C'est ce que je veux dire quand je parle de regard érotique. Ce que je vois n'est plus alors simple objet de perception, mais part intime de moi-même. Autrefois je pense qu'on appelait cela intuition des essences. Un détail isolé devient signe de l'ensemble. Ce que j'écris n'est plus dès lors ce que j'ai observé, comme c'est le cas pour la plupart, mais ce que j'ai vécu. C'est justement pour cela que je veux devenir écrivain. (FB, 58)

5 Intuition, révélation, «heure de la sensation vraie », telle que la vit Keuschnig sur le banc au Carré Marigny (SwE, 81) : ce sont ces moments qui rendent possible l'autre regard, qui lui, fonde à présent pour Handke le projet d'écriture. Et si celle-ci s'est tant modifiée depuis le style laconique des premiers récits, c'est qu'elle est à la recherche des mots pour dire cette perception nouvelle. Encore est-il inexact de parler ici de perception, qui se définit par son objet et qui a affaire avec les lois de l'optique. C'est cette dimension du VU qui détermine ce rapport purement extérieur aux images dont souffre Bloch dans L'Angoisse du gardien de but au moment du penalty, fait de fascination et de frontalité. La perception met en œuvre la surface et non la pénétration. Le sujet est en marge, comme ce curieux voyageur de La courte lettre pour un long adieu traversant un paysage américain qui lui reste étranger, qui est mis à distance par le cadrage de la vitre de motel, du pare-brise, du rétroviseur, Amérique vue en vignettes et où i n'a pas sa place. La perception peut aussi s'exaspérer, vouloir tout voir, par déception de ne rien saisir, comme dans les romans de Robbe-Grillet, comme pour Bloch. Le désir de panopticité veut tout découvrir, et tout devient, d'être objet de convoitise, lieu de mystère et de perversité. L'autre regard au contraire ouvre une dimension toute différente : il n'a pas affaire avec le VU, ni le VISUEL, mais le VISIBLE. Le Vu s'oppose au non-vu, au caché, au honteux: dans L'heure de la sensation vraie Keuschnig en fait, jusqu'au moment de la révélation, mille fois l'expérience. Voir et avoir honte sont pour lui presque simultanés. Le VISIBLE s'oppose lui à l'invisible : et alors que le non-vu se divise et se nomme, l'invisible ne se nomme pas, il est le " contexte ", la somme de tout le visible qui ne se donne dans aucune addition. Le visuel peut se formuler selon les lois de la géométrie, que maîtrise Sorger, le géologue du Lent retour. C'est aussi l'espace de la circulation des signes, du glissement des surfaces, du «télévisuel » que Handke analyse et accuse dans Chronique des événements courants. Le visible est de l'ordre du sensible, de cette sensation qui est vérité, parce qu'elle n'est pas exclusion du sujet percevant, mais échange, "érotisme » : l'objet s'inscrivant dans le sujet, et le sujet dans l'objet. Or de tels instants de vrai regard sont rares, exceptionnels. Les personnages de Handke se mettent en quête de leur possible continuité, et en ce sens ils ne font que reprendre dans leur histoire le problème de celui qui la raconte, l'écrivain Handke. Les livres, à partir de Lent retour sont souvent des parcours dans l'espace, promenades, pèlerinages, au rythme des pas, et Georges-Arthur Goldschmidt, le traducteur de Handke, insiste 
justement sur cette expérience physique de la spatialité5. Mais l'écriture est elle aussi en chemin, cherchant à se frayer un passage, retombant parfois dans des moments d'incertitudes, désirant la VISION qui lie le visible. Sorger rêve d'un « répertoire de tous les espaces ", ceux de la géographie comme ceux de l'imaginaire ; Loser dans Le Chinois de la douleur médite sur la notion de seuil, qui est moins rupture que transition, qui donne forme en dissociant et unissant, figure paradoxale de la continuité. Tous ces récits pourtant ne parviennent pas à faire oublier cette nuance d'irréalité qu'ont les épiphanies.

6 La solution, s'il en est une, est apportée par un petit texte La Leçon de la Sainte-Victoire. Handke y raconte deux ascensions à la montagne de Cézanne, mais y parle aussi des rapports de l'art et du réel, du récit et de la sensation vraie, de l'espace parcouru et de l'espace du tableau. En ce sens ce petit livre est une sorte de poétique, juste esquissée, aérienne. En son centre se situe la révélation de la peinture de Cézanne. Non pas une épiphanie de plus, subjective et évanescente, mais la réalité du «retournement perceptif », le tableau étant là pour en donner la preuve, tangible, permanente. Une preuve qui est à l'épreuve du temps, qui trouve une miraculeuse réponse au problème de la Durée :

Je fis l'expérience de ce bond que font deux regards séparés par l'intervalle du

temps pour se rencontrer sur la surface d'un même tableau. (LSV, 38)

7 s'écrie le narrateur-écrivain devant une toile de Cézanne. Le regard de Cézanne est plus que le regard d'enfant ou que le regard tiers, tel celui de John Ford à la fin de La courte lettre pour un long adieu: il n'est pas singularité d'une perception, ni promesse d'un ordre autre, il est vision de l'essence même de l'espace, où s'abolit l'écart entre le Dehors et le Dedans, entre le vécu et le réel, entre le Moi et le Monde. Cézanne fait non seulement comprendre, mais voir à Handke comme autrefois à Rilke la réalité de la cohésion spatiale. Ce que Sorger ne faisait que pressentir : «Le lien est possible » (LH, 112), l'écrivain peut à présent en être certain (LSV, 100).

8 La dimension ontologique de La Leçon de la Sainte-Victoire est affichée. Pour la première fois être et représentation sont intimement liés. Handke traverse au Tholonet à la fois le paysage réel et le paysage peint, et la peinture donne forme à la spatialité vécue dans le corps, c'est à Paul Cézanne qu'il « doit d'être dans les couleurs du jour » (LSV, 16). Ainsi se réalise l'utopie de la " géographie enfantine » de Sorger : être dans les couleurs signifie être au monde, non pas tant y être présence qu'être dans sa présence. La couleur, que Handke avoue ne pas toujours bien distinguer, non par un défaut physique, mais par manque de foi, cette couleur est la substance charnelle des choses existantes, elle est à la fois en elles et dans le regard, elle est le lieu même de leur "entrelacs» (selon le mot de Merleau-Ponty cherchant à percer le mystère de la peinture de Cézanne'). Handke rappelle ce phénomène dont parle Stifter dans ses souvenirs, cette fusion primitive dans la couleur :

Il y avait en moi des taches sombres. Le souvenir m'apprit plus tard que c'était des

forêts. (LSV, 14)

L'importance de l'image picturale est un fait nouveau : certes on voyait, dans la scène finale du récit La Femme gauchère, Marianne dessiner patiemment ce qui l'entourait. Certes Sorger le géologue est aussi dessinateur. Mais l'espace pictural est davantage que le trait, et Cézanne parvient à ce qu'il nomme la "réalisation" en abandonnant justement la méthode du cerne. Le dessin (et le cinéma encore davantage) ne crée pas cette spatialité qui englobe spectacle et spectateur: l'objet y est toujours comme vu de 
l'extérieur. S'interrogeant sur son rapport aux images de la peinture, Handke tente d'en relater l'évolution. Et il constate qu'elles lui furent d'abord étrangères. Lorsqu'il était enfant, dans son village de Carinthie, elles étaient les ex-votos dans la montagne, les images pieuses à l'église, simples illustrations d'une signification qui les outrepassait. Elles étaient "accessoires » et il "n'en attendait rien de décisif » (LSV, 16). Il lui semblait qu'elles détournaient plutôt de l'essentiel et que le vide d'images était mieux fait pour exprimer "cette vibration infinie entre l'âme et Dieu » (LSV, 17) que pourrait être le sentiment religieux. Plus tard, quelques toiles entrevues deviennent avec le temps "des images de vie et de rêve", des images magiques : ce sont les places métaphysiques de Chirico, les Villes de Max Ernst, l'Empire des Lumières de Magritte. Espaces nus, vidés de toute présence humaine, dans un énigmatique suspens entre veille et sommeil, entre bonheur et menace. Les lieux oniriques de Chirico, les constructions abstraites de Max Ernst, les montages intelligents et rêveurs de Magritte mettent en place une spacialité abstraite, une topologie précise et toute occupée d'opérations mentales, mais qui n'est pas celle de l'habiter. On retrouve-là un peu de cette atmosphère prête à se teinter de cauchemar qui baigne les premiers textes de Handke. C'est ensuite la découverte d'Edward Hopper : ses tableaux sont tout aussi magiques que les toiles des surréalistes, mais ils restent en-deçà de la frontière du rêve, ils se tiennent à l'exacte limite entre réel et irréel: les petites maisons de Cape Cod existent bien vraiment, Handke est allé les voir, et l'une d'entre est devenu la maison de Sorger dans Lent retour (LH, 93). Et pourtant elles sont "une réalité à l'abandon ", vibrant dans une sorte d'attente silencieuse. Les lieux de Hopper servent, avant ceux de Cézanne, de pèlerinage. Et, comme plus tard à la montagne Sainte-Victoire, Handke a la sensation d'être dans l'espace du tableau :

Lorsqu'il y a quelques années j'allais sur les traces de ses tableaux à Cape Cod, qui m'attirait depuis longtemps déjà, j'eux pour la première fois l'impression, partout sur cette langue de terre, d'être dans l'empire d'un artiste. Je pouvais à présent suivre les courbes, les élévations et les abaissements de la route de dunes. Les détails, souvent étrangers aux toiles d'Hopper, se trouvent dans ma mémoire à droite et à gauche, comme sur un tableau. Au beau milieu d'une de ces « imagesposthumes ", l'épi d'un roseau planté dans la glace épaisse, équilibré par la boite de conserve qui traîne à côté. (LSV, 19)

10 L'admiration pour Courbet peut étonner de la part de Handke. Il est avant tout sensible à la qualité de silence de ces toiles qui parlent si peu du peintre et laissent toute place au motif :

Je fus saisi par le silence souverain de ces tableaux... Et je sus que c'était les images justes, justes pour moi, mais pour les autres aussi. (LSV, 31)

11 Handke rappelle que Cézanne tenait La grande vague de Courbet pour « la découverte du siècle » (LYS, 33). Il y a dans La Leçon de la Sainte-Victoire une « découverte » semblable : celle d'un tableau intitulé Rochers près des grottes au-dessus de Chateau-Noir, que Cézanne a peint en 1904 et que Handke voit au Musée du Jeu de Paume : reprenant la métaphore rilkéenne du vol d'oiseau, il tente de décrire la sensation qui se produit-là, celle de la totale cohésion du sensible :

... les pins et les rochers se dressaient tels quels en moi, comme fait l'oiseau qui s'envole et traverse notre corps le temps d'un instant à grands coups d'aile, mais sans disparaître, comme dans ce moment d'épouvante, en restant là. (LSV, 76)

12 Ce que Gerhard Melzer appelle « la phénoménologie du regard » chez Handke ${ }^{7}$, marque à la fois l'originalité de son projet d'écriture et ses limites-même: le modèle de la peinture est bien ambigu. Car d'une part il fonde la légitimité de l'autre regard, tout en 
constituant pour le récit comme un point de butée : ce que le tableau peut montrer, dans la souveraineté de son silence, dans l'éternité d'un instant suspendu, la dynamique inhérente à toute narration l'interdit. Handke le reconnaît, et il n'évite par l'écueil, dans La Leçon de la Sainte-Victoire il le réfléchit au contraire, comme le montrent par exemple les digressions à propos de l'inévitable tension des récits de Stifter, qui pourtant ne voulait rien d'autre que de dire «la douce loi de la Nature» (LSV, 74). Le texte lui-même ruse, module ses rythmes, introduisant la distance, ralentissant la progression, jouant davantage sur le vide que sur le plein. D'autres récits, Lent retour, Le Chinois de la douleur, Le Recommencement sont contraints d'accepter comme leur loi cette étrange oscillation entre mystique et connaissance, qui, le note Michel de Certeau, est le propre de l'acte de voir, mais appartient moins au dire :

Le sujet voyant est un être particulier qui vise dans ce qu'il distingue la réalité même dont il se détache. Les objets qu'il voit composent donc la représentation de ce dont il a du se séparer pour le connaître. Ils lui racontent une solidarité brisée une origine perdue - et un retour vers les choses par les chemins de la conscience. Ils lui sont donc la légende de sa propre histoire (la légende: ce qu'il faut lire, legendum), une histoire sans paroles dont la vision est déjà la pensée, jouant dans l'écart qui fait des choses vues à la fois des objets vers lesquels la connaissance va et des signes, quasi mythiques, de la réalité d'où elle vient ${ }^{8}$.

L'écriture de Handke se trouve exactement dans la position décrite ici par Michel de Certeau: prise entre la tentation du silence (qui explique la fascination pour des peintres comme Hopper, Courbet et Cézanne) et la nécessité de faire des objets de la vision (et non plus la perception), des objets mythiques. Ainsi l'arbre, véritable motif de La Leçon de la Sainte-Victoire: le Grand Pin, motif de Cézanne d'abord, les pins parasol de la Provence, le mûrier entrevu au fond d'un chemin creux et qui fait écho à un autre mûrier, aux fruits rouges tombés, tachant le sol, des années auparavant, sur la côte dalmate (LSV, 23, 25, 28, 72). L'arbre est la figure d'un moment de communion, mais dans l'instant même de son évocation, il est donné comme perdu. La déchirante "séparation", «l'origine perdue », «la solidarité brisée» sont nécessairement contenues dans cette écriture, qui pourtant s'applique à les dépasser.

L'écriture pour Handke est cette lente activité qui donne des mots à un regard. Et c'est en vérité une activité de poète. Handke «travaille» la prose pour lui donner la transparence du verbe poétique : elle lui résiste parfois, et dans un texte long comme celui du Recommencement, le lecteur reconnaît aisément les passages où la résistance a été la plus forte. Kobal, le personnage du roman, est d'ailleurs à la recherche de la langue de ses origines, un slovène à demi-oublié et dans lequel il pense retrouver une pureté que l'autre langue a perdue, une proximité des choses, dont la lisibilité, elle, n'est plus mise en doute. Parlant du Recommencement, Handke précise :

[Dans ce récit]... il se produit une oscillation permanente entre les objets du monde et leur lent passage à l'écrit, de telle sorte que tout ce que le héros perçoit, dans sa succession même, lui apparaît comme une écriture que l'on devrait faire émerger. $(\mathrm{Zr}, 231)$

L'émergence des choses dans le langage, le "déchiffrement ", la recherche d'une écriture qui soit entièrement trace : voilà le but à atteindre. Or Handke, et c'est là la spécificité de ses textes, met en scène à la fois le projet et sa réalisation. Depuis Le Chinois de la douleur, comme le note Christoph Bartmann, tous ses livres pourraient porter le titre de l'un d'entre eux: Après-midi d'un écrivain. Le fragment réalise l'idéal, qui est un pur dire du réel : 
Les fleurs blanches des fraisiers, vascillant sous les gouttes de la pluie (de la fenêtre du ravin) ${ }^{9}$.

Le récit, lui, est pris entre géo-graphie et auto-graphie, et cette tension constitue sa véritable dynamique : il est d'abord regard (le lecteur garde en mémoire des images précises et pourtant rêveuses, paysages réels et lieux intimes à la fois), tout en étant le désir répété de " raconter » ce regard. Et cette histoire qui aurait exclu le Mal et la Mort serait ce que Handke appelle « une épopée de la paix ».

Une épopée faite de haikkus mais qu'on ne remarquerait nullement en tant qu'objets isolés, sans action, sans intrigue, sans drame, et qui pourtant raconterait : c'est ce que j'entrevois comme le but suprême. (GB, 52)

\section{ANNEXES}

\section{Abréviations}

AdT : Die Angst des Tormanns beim Elfmeter, Suhrkamp, Francfort, 1970.

FB: Falsche Bewegung, Suhrkamp, Francfort, 1975.

GB: Die Geschichte des Bleistifts, Residenz, Salzbourg, 1982.

H: Die Hornissen, Suhrkamp, Francfort, 1966.

kB: Der kurze Brief zum langen Abschied, Suhrkamp, Francfort, 1972. LH: Langsame Heimkehr, Suhrkamp, Francfort, 1979. LSV: Die Lehre der Sainte-Victoire, Suhrkamp, 1981.

$\mathrm{Zr}$ : Aber ich lebe nur von den Zwischenräumen - Ein Gespräch mit Herbert Gamper, Ammann, Zurich, 1987.

\section{NOTES}

1. - P. Klee, Schöpferische Konfession - Das bilderische Denken, Berne, 1956, p. 76.

2. - Voir à ce propos Jürgen Becker, «Peter Handkes erster Roman Die Hornissen» in Michael Scharang, Uber Peter Handke, Suhrkamp, 1972, p. 19.

3. - Les quatre romans des années soixante-dix sont d'une écriture très cinématographique et ont été tous les quatre portés à l'écran :

Die Angst des Tormanns beim Elfmeter, 1970, adapté par Wim Wenders en 1972.

Der kurze Brief zum langen Abschied, 1972, adapté par Herbert Vesely pour la télévision, en 1978.

Die Stunde der wahren Empfindung, 1976, adapté par Didier Goldschmidt sous le titre Ville étrangère, 1988.

Die linkshändige Frau, 1976, adapté par Handke en 1977.

A propos de la facilité de l'adaptation voir Wim Wenders in Cahiers du cinéma, n400, p. 47. 
4. - Georges-Arthur Goldschmidt, «Raumglück?» in Die Arbeit am Glück - Peter Handke, édité par Gerhard Melzer et Jale Tükel, Athenäum, 1985.

5. - M. Merleau-Ponty, Le Visible et l'Invisible, Gallimard, 1964, p. 54.

6. - Gerhard Melzer, «Lebendigkeit: ein Blick genügt - Zur Phänomenologie des Schauens bei Peter Handke», in Die Arbeit am Glück, op. cit., p. 126.

7. - Michel de Certeau, revue Esprit, juin 1982, p. 92.

8. - Christoph Bartmann, «Das Gewicht der Welt - revisited», in Text und Kritik, ${ }^{\circ}{ }^{\circ} 24$, novembre 1989, p. 34.

9. - «Am Felsfenster, morgen», notes parues dans Manuskripte, n97, octobre 1987, p. 9.

\section{RÉSUMÉS}

Les premiers textes de Peter Handke sont comme constitués de pans de visuel, organisés en un montage lâche d'images autonomes. Le personnage, lorsque celui-ci est mis en scène, subit davantage qu'il ne maîtrise le défilement de ces images agressives, faites pour moitié de perceptions d'un espace morcelé, pour moitié de souvenirs surgissant bruts d'une mémoire malheureuse, de scènes lues, de fragments de films. La métaphore de l'aveugle, récurrente dans les premiers récits, exprime l'absence de regard et la tout-puissance d'images sans sujet. À partir de L'angoisse du gardien de but au moment du penalty se constitue progressivement un lieu du regard, comme intuition de la cohésion spatiale d'abord, puis comme conviction de l'unité du Sensible. Dans La Leçon de la Sainte-Victoire, Handke parle de son rapport à la peinture et dit sa découverte de Cézanne dont les tableaux donnent à voir une spatialité qui inclut corps et paysage, réel et imaginaire. C'est dans cette dimension que Handke veut placer son écriture.

Das visuelle Moment kennzeichnet Handkes Frühprosa: Der Text ist nichts als eine lose Montage von Bildern, die für sich stehen. Die Romanfigur - wenn es überhaupt eine gibt - ist ihnen wehrlos ausgeliefert. Es sind bruchstückhafte Raumerfahrungen, Fragmente einer Realität, die wie unter der Lupe wahrgenommen wird, Erinnerungen schmerzhafter Erlebnisse, die urplötzlich aus dem Gedächtnis auftauchen, aber auch Erinnerungen an einst gelesene Bücher oder an Filmszenen. Erst allmählich wird das Subjekt Herr über die Bilderflut, die Ausdruck der Welt- und Selbstentfremdung ist. Seit Die Angst des Tormanns beim Elfmeter ist eine Blickrichtung in Handkes Texten erkennbar, die zuerst bestimmt wird durch die intuitive Erfassung des räumlichen Zusammenhangs (Die Stunde der wahren Empfindung) und dann durch den Glauben an eine Zusammengehörigkeit der Dinge, wie sie sich zum Beispiel in Cézannes Gemälden offenbart. Die Lehre der Sainte-Victoire ist das Bekenntnis zu diesem neuen Weltgefühl, welches zugleich neues Raumerlebnis und neue Dimension des Schreibens ist.

\section{AUTEUR}

\section{ARLETTE CAMION}

Université Charles-de-Gaulle - Lille III 